\title{
Numerical Simulation Of Heat Transfers Synergistic Performance Of He/S-CO2 Mixture Gas With The Heat Transfer Enhancement Structure On The First Wall Of Fusion Reactor
}

Ji junyu

Anhui University

Wang Xiaoyu

Southwest Institute of physics of nuclear industry

Zhou weibin

Anhui University

Gao jun

Anhui University

Lu Wei

Anhui University

Chu delin

Anhui University

Wang Weihua ( $\nabla$ whwang@ipp.ac.cn )

Anhui University

\section{Research Article}

Keywords: heat transfer enhancement, binary mixture gas, first wall, fins,

Posted Date: February 28th, 2022

DOI: https://doi.org/10.21203/rs.3.rs-1394278/v1

License: (c) (1) This work is licensed under a Creative Commons Attribution 4.0 International License.

Read Full License 


\title{
Numerical simulation of heat transfers synergistic performance of $\mathrm{He} / \mathrm{S}-\mathrm{CO}_{2}$ mixture gas with the heat transfer enhancement structure on the first wall of fusion reactor
}

\author{
Ji junyu $^{\mathrm{a}}$, Wang xiaoyu ${ }^{\mathrm{b}}$, Zhou weibin $^{\mathrm{a}}$, Gao jun ${ }^{\mathrm{a}}, \mathrm{Lu} \mathrm{Wei}^{\mathrm{a}}$, Chu delin ${ }^{\mathrm{a}}$, Wang weihua ${ }^{\mathrm{a} *}$ \\ a. Anhui University, Hefei, 230031, China; \\ b. Southwest Institute of physics of nuclear industry, Chengdu 610041, China
}

\begin{abstract}
As a new cooling medium, Helium/ Supercritical Carbon Dioxide $\left(\mathrm{He} / \mathrm{S}-\mathrm{CO}_{2}\right)$ binary mixture gas can effectively improve the heat transfer capability. In this paper, the heat transfers synergistic performance of $\mathrm{He} / \mathrm{S}-\mathrm{CO}_{2}$ with fins structure were numerically simulated by ANSYS / CFD method. The results show that the pressure drop in the channel decreases gradually with the increase of volume fraction of $\mathrm{S}-\mathrm{CO}_{2}$. Comparing with smooth channel, fin structure can significantly improve the intensity of convective heat transfer intensity, the average Nusselt number of flow channel with finger fins are increased by $80 \%$. The heat transfer efficiency of $\mathrm{He} / \mathrm{S}-\mathrm{CO}_{2}$ binary mixture gas coupled with heat transfer enhancement structure (triangular fins) is increased by $310 \%$ at the condition of volume ratio of $\mathrm{S}-\mathrm{CO}_{2}$ to He with $2: 1$. Further study on the optimized scheme of first wall (FW) of International Thermonuclear Experimental Reactor (ITER) China Helium-Cooled Ceramic Breeder-Test Blanket Module (HCCB-TBM) show that triangular fins are placed on the inner surface of $\mathrm{FW}$ faced deuterium-tritium plasma, and the optimized distance between fin pitches is $7 \sim 10 \mathrm{~mm}$. The results can provide
\end{abstract}


meaningful references for the cooling scheme design of the FW in ITER. Keywords: heat transfer enhancement; binary mixture gas; first wall; fins;

\section{Introduction}

The first wall (FW) of the breeder blanket of fusion reactor needs to bear the high heat flux and neutron radiation from the core plasma. To ensure the safe operation of the device, the technology of efficient cooling is one of the key challenges in the design of fusion reactor. Due to its excellent characteristics of good chemical inertia and low neutron absorption cross section [1,2], helium (He) is widely used as coolant in the design scheme of breeder blanket. Meanwhile, for the low density, small specific heat capacity and low heat exchange efficiency of helium, higher pump power is required to cool structural materials, for example RAFM steel with the operating temperature range between 300 and $550{ }^{\circ} \mathrm{C}$, which inevitably increases the engineering complexity and reduces the net output power of the reactor.

The thermophysical and transport characteristics of supercritical carbon dioxide (S$\left.\mathrm{CO}_{2}\right)$ change significantly near the critical point $\left(31^{\circ} \mathrm{C}, 7.38 \mathrm{MPa}\right)$, which has a great impact on turbulent heat transfer[3]. Due to its unique energy transmission characteristics

This document is the results of the research project funded by the National Natural Science Foundation of China under Grant 11975022 and in part by the National Magnetic Confinement Fusion Program under Grant2017YFE0300603, Grant2018YFE0309100, and Grant2015GB120006.

* Corresponding author

Email address: whwang@ipp.ac.cn 
and low transmission power requirements, $\mathrm{S}-\mathrm{CO}_{2}$ as a cooling medium shows excellent performance in terms of cycle efficiency, equipment cost and tritium permeating resistance [4,5]. Kato, et. al., [6] found that the cycle efficiency of $\mathrm{S}^{-\mathrm{CO}_{2}}$ as the cooling working medium of gas cooled reactor is about 3-9\% higher than that of traditional Helium cooling cycle. Relevant studies show that the binary mixture produced by mixing S-CO2 and helium can significantly reduce the critical point of $\mathrm{CO}_{2}$ and significantly improve the cycle efficiency [7].

The allowable temperature of blanket structural material (RAFM steel) is an important factor limiting the thermal load bearing capacity. In order to efficiently cool the high heat flux of FW, Cheng [8] designed the heat transfer enhancement schemes by roughening the inner wall surface of the helium flow channel, setting fins and other enhanced heat transfer methods, or enhancing the turbulence disturbance type into a secondary vortex in the flow boundary layer. Andreozzi[9] studied three types of heat transfer enhancement structures (triangular fins, rectangular fins, trapezoidal fins) applied to tubular heating channels, and found that the triangular fins has the best heat transfer performance but higher pressure drop. Hou, et. al.,[10] arranged a rectangular heat transfer enhancement structure on the surface of the wall for the experimental device. The critical heat flow (CHF) can be increased to more than $80 \%$ compared with the smooth flow channel.

The existing convective enhanced heat transfer technology is mainly to install fins, increase roughness and grooves on the smooth wall surface to enhance the turbulence 
intensity by strengthening the flow field disturbance and the convective heat transfer near the wall surface. Until recently there is still little about the synergistic performance of binary mixture gas with enhanced heat exchange structure to further improve the heat transfer capacity and efficiency. This paper proposes a new method of co-cooling FW with helium as the base gas, mixed with $\mathrm{S}-\mathrm{CO}_{2}$ to form a binary mixture gas and synergistic heat transfer with fins structure. The composition effects of binary mixture gas and the structure of fins on the heat transfer performance are numerically analyzed by ANSYS / CFD method.

\section{Calculation model and boundary conditions}

Fig. 1(a) shows the main design parameters of Helium-Cooled Ceramic Breeder Test Blanket Module (HCCB-TBM) of China. Fins structure is arranged on the inner surface of FW facing the plasma side (Fig.1(b)). Some of the most promising and effective fin structures like rectangular fins, triangular fins, finger fins, are presented here (Fig.1(c)). The channel length is $350 \mathrm{~mm}$. In order to reduce the amount of calculation and considering the symmetry of the model, only a single flow channel model is established. 
(a)

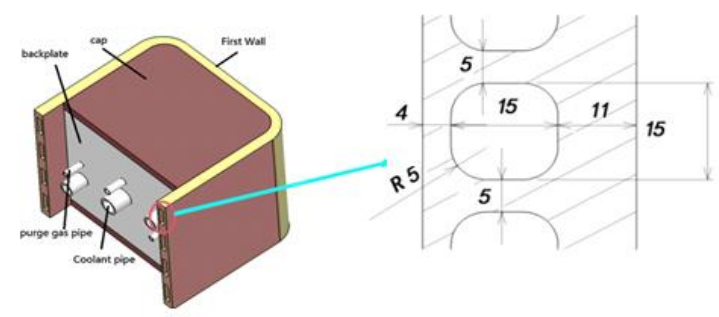

(b)

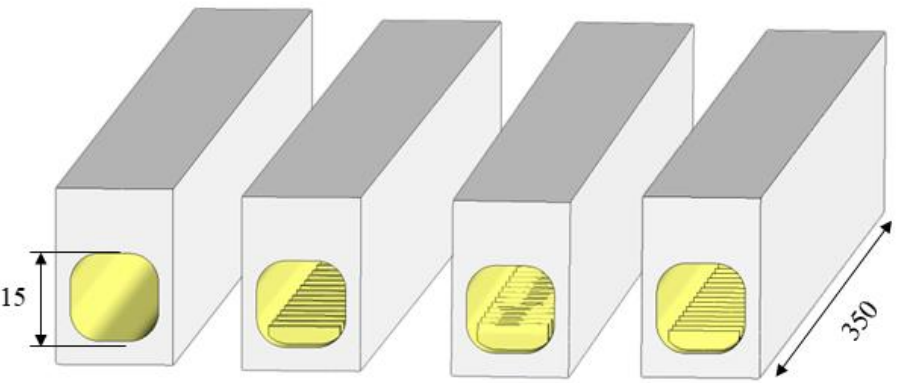

(c)

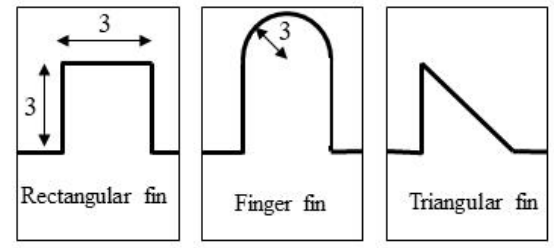

Fig.1 (a) HCCB-TBM model. (b) Geometrical model with different fin. (c) Different shapes of fin

For the complex heat transfer, the temperature interface fluctuates greatly at the gassolid. In order to obtain better calculation accuracy, mesh encryption is carried out at flow boundary. The sensitivity comparison analysis of the numerical grid is checked out in the calculation. The boundary layer grid division and calculation convergence accuracy of the fluid meet the numerical requirements. Chemical reaction doesn't exist between $\mathrm{S}-\mathrm{CO}_{2}$ and $\mathrm{He}$ gas during heat exchange. The mixed gas is defined by Species Transport model, and the boundary condition parameters are shown in Table 1 .

Table 1 boundary condition parameters

\begin{tabular}{cc}
\hline Parameter & Value \\
\hline Heat flux & $0.4 \mathrm{MW} / \mathrm{m}^{2}$ \\
Structural materials & RAFM steel \\
Coolant & $\mathrm{He} / \mathrm{S}-\mathrm{CO}_{2}$ with different volume ratios
\end{tabular}




\section{Material physical parameters and evaluation criteria}

The variation of physical properties of $\mathrm{He}$ and $\mathrm{S}-\mathrm{CO}_{2}$ with temperature under $8 \mathrm{MPa}$ are considered in the numerical simulation. Compared with $\mathrm{He}, \mathrm{S}-\mathrm{CO}_{2}\left(7.37 \mathrm{MPa}, 31^{\circ} \mathrm{C}\right)$ has higher density, lower thermal conductivity, specific heat capacity and viscosity. The effect of temperature change on the density of RAFM steel is small and can be ignored. The physical parameters of $\mathrm{He} / \mathrm{S}-\mathrm{CO}_{2}$ mixture is defined by the following formula.

Density of gas mixture:

$$
\rho=\frac{1}{\sum_{i} \frac{m_{i}}{\rho_{i}}}
$$

The thermal conductivity is the mass average of the thermal conductivity of each component:

$$
k=\sum_{i} m_{i} k_{i}
$$

The calculation of viscosity is based on the average value of single component viscosity:

$$
u=\sum_{i} m_{i} u_{i}
$$

The specific heat capacity is defined as the mass average of the specific heat capacity of each component:

$$
c_{p}=\sum_{i} m_{i} c_{p, i}
$$

The cooling performance of the flow channel heat sink with the different fins is evaluated using conventional heat transfer quantities. The local convective heat transfer coefficient $(h)$ and Nusselt number $(\mathrm{Nu})$ are defined as: 


$$
\begin{gathered}
h=\frac{q}{T_{w}-T_{m}} \\
N u=\frac{h D_{h}}{k}
\end{gathered}
$$

Where: $\mathrm{D}_{h}$ is the hydraulic diameter, $q$ is the heat flux, $\mathrm{T}_{\mathrm{w}}$ is the average temperature of the heating surface of the solid inner wall, $\mathrm{T}_{\mathrm{m}}$ is the bulk temperature of fluid

A Figure of Merit (FOM) is used to evaluate the overall thermohydraulic performance by considering economy and operation efficiency $[11,12]$. It is determined as:

$$
F O M=\frac{N u / N u_{0}}{\left(f / f_{0}\right)^{\frac{1}{3}}}
$$

The criterion considers the increase of resistance caused by enhanced heat transfer coefficient. Therefore, it can be considered that the larger the FOM performance coefficient, the better the comprehensive heat transfer performance.

The calculation expression of the average friction factor $f$ is defined as:

$$
f=\frac{2 D_{h} \Delta p}{\rho u_{i n}^{2} L}
$$

Where: $\Delta p$ refers to inlet and outlet pressure difference, $\rho$ refers to fluid density and $\mu_{i n}$ refers to average flow velocity.

\section{Results and discussions}

\subsection{Surface wall temperature and pressure drop of the smooth flow channel}

With the volume fraction alteration of $\mathrm{S}-\mathrm{CO}_{2}$ in the binary mixture gas, the variation curves of the FW maximum temperature and pressure drops in the smooth flow channel 
are illustrated in Fig. 2, respectively. As shown in Fig.2, the maximum temperature of the FW gradually rises with the increase of volume fraction of $\mathrm{S}-\mathrm{CO}_{2}$, and the variation tendencies of pressure drops in flow channels are completely opposite. This is mainly because $\mathrm{He}$ presents higher thermal conductivity than $\mathrm{S}-\mathrm{CO}_{2}$. With the increase of $\mathrm{S}-\mathrm{CO}_{2}$ in the binary mixture gas, the thermal conductivity of the binary mixture gas gradually decreases and the ability of fluid to transfer heat becomes weak. On the other hand, due to the doping of macromolecular $\mathrm{S}-\mathrm{CO}_{2}$, the density of binary mixture gas gradually increases. The fluid flow rate decreases in the same condition, so the flow channel pressure drop decreases.

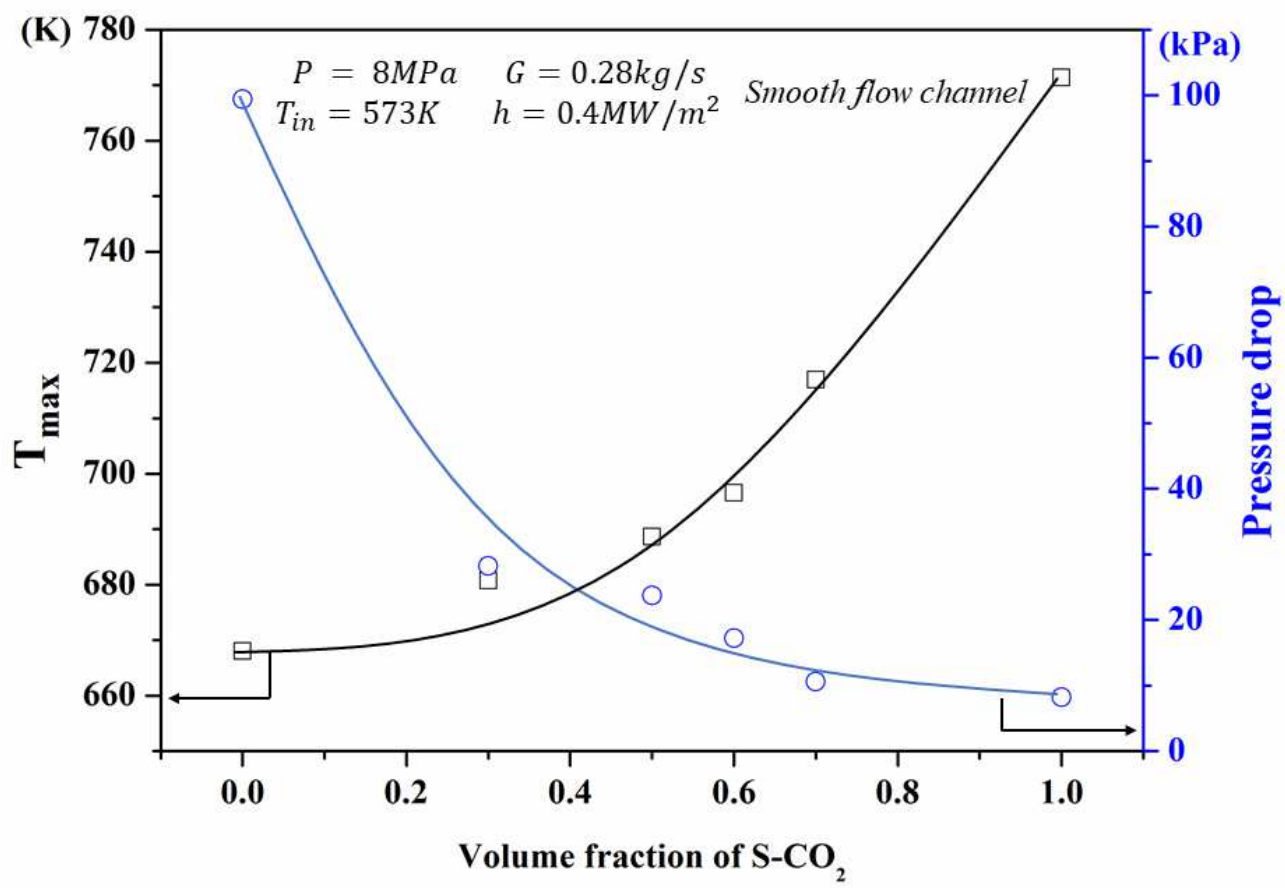

Fig.2. The maximum temperature of FW and pressure drops with changing of the volume fraction of $\mathrm{S}-\mathrm{CO}_{2}$ in the binary mixed gas

Fig. 3 is the temperature distribution at the $200 \mathrm{~mm}$ cross-section of the flow channel 
with different volume ratios of $\mathrm{He} / \mathrm{S}-\mathrm{CO}_{2}$ under the same conditions of mass flow rate. It indicates that in the flow channel, the fluid temperature stratification obviously occurs at the region which is nearer to the heated surface. The fluid in the central of channel does not fully transfer heat to the wall, thus has the lowest temperature. The temperature difference increases gradually with the increase of $\mathrm{S}-\mathrm{CO}_{2}$ ratio and the maximum temperature difference reaches $30 \mathrm{~K}$ at the fluid working medium is $\mathrm{S}^{-\mathrm{CO}_{2}}$ (as shown in Fig. 3e).
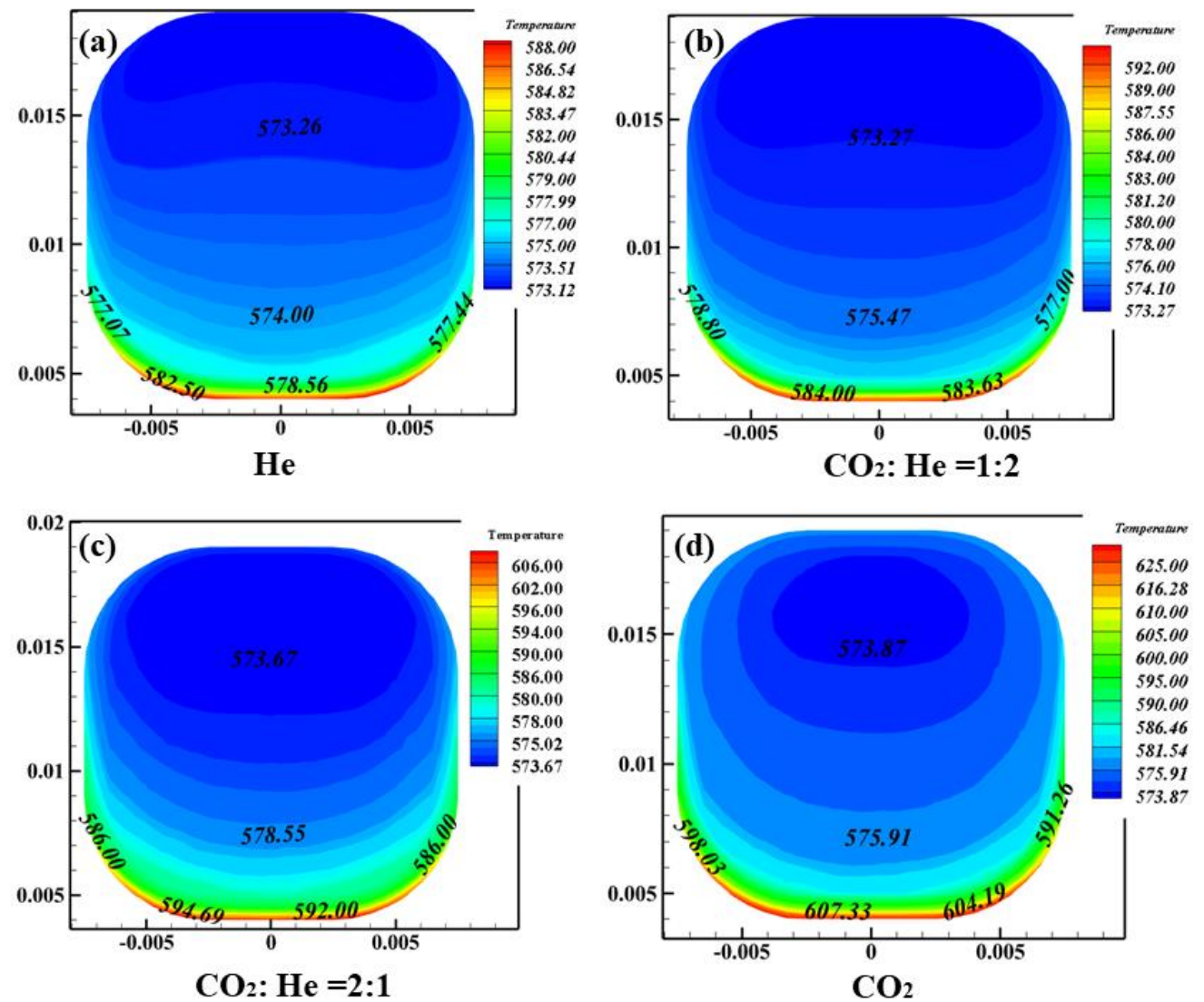

Fig. 3 Temperature distribution at $200 \mathrm{~mm}$ cross-section of flow channel (K) with different volume ratios of $\mathrm{He} / \mathrm{S}-\mathrm{CO}_{2}$ under the same conditions of mass flow rate

\subsection{Heat transfer characteristics of Helium with different fins}

Fig.4 shows the temperature distribution in the $\mathrm{CuCrZr}$ tube under helium cooling. 
The allowable temperature of $\mathrm{CuCrZr}$ material under the working condition are $723 \mathrm{~K}$. As shown in Fig. 4(a), the temperature of smooth flow channel is lower near the heating surface at the entrance, and then the surface temperature increases rapidly, reaching the maximum of 667.4 K. Fig.4 (b) (c) (d) show the temperature distribution for the $\mathrm{CuCrZr}$ tube with rectangular fins, finger fins and triangular fins distribute relatively at the temperature of $664 \mathrm{~K}, 659 \mathrm{~K}$ and $659.1 \mathrm{~K}$ respectively. It means the fin structure can transfer more heat flux away from wall surface, and different fin structures cause different heat transfer enhancement effects.
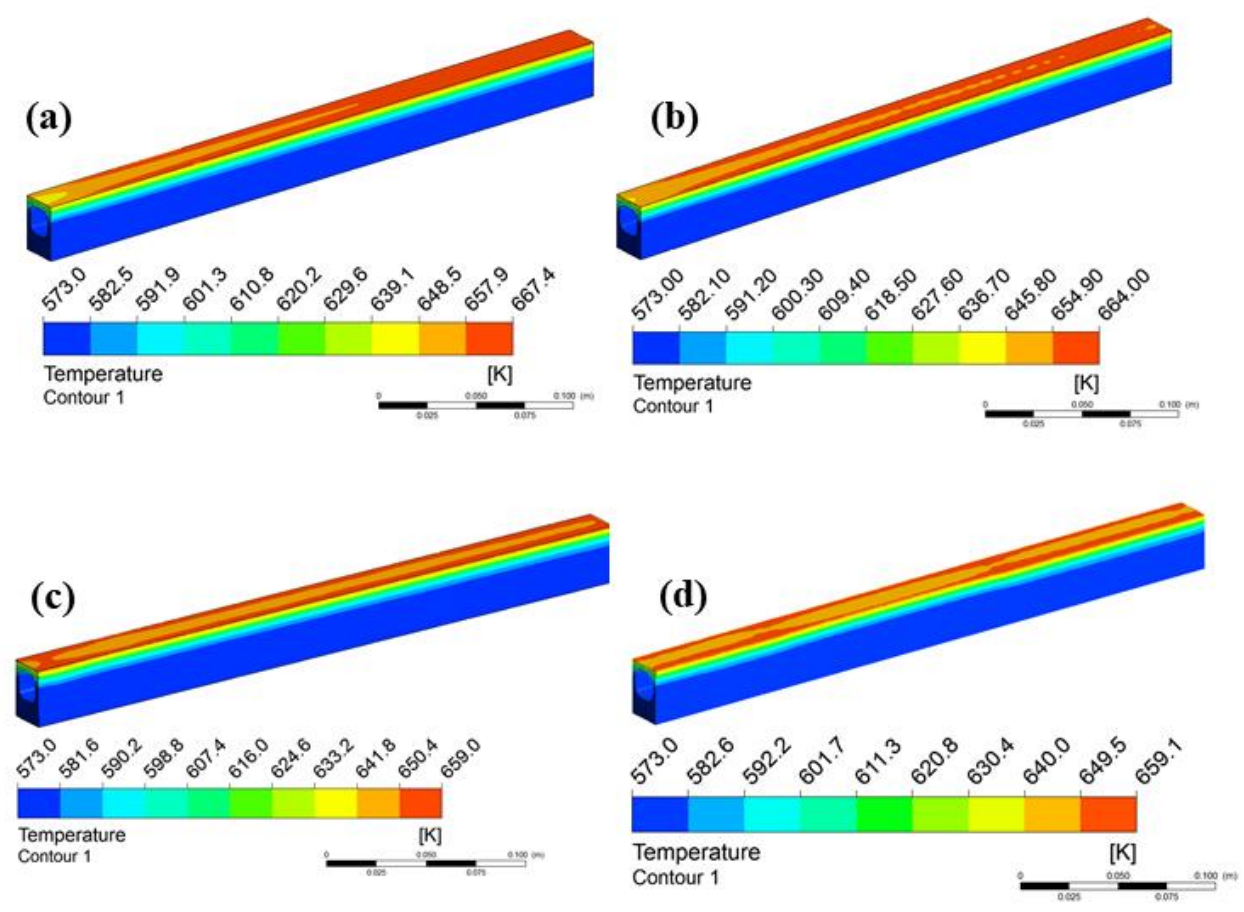

Fig.4 Temperature distribution for the $\mathrm{CuCrZr}$ tube at $0.4 \mathrm{MW} / \mathrm{m}^{2}$ when $\mathrm{G}=0.28 \mathrm{~kg} / \mathrm{s}$, $\mathrm{T}_{\text {in }}=573 \mathrm{~K}, \mathrm{P}=8 \mathrm{MPa}$ (a) smooth flow channel (b) flow channel with rectangular fins (c)flow channel with finger fins (d) flow channel with triangular fins

The temperature distribution of He on the cross section of the channel $(x=0, z=0.45$ $\sim 0.48 \mathrm{~mm}$ ) is further analyzed. As shown in Fig. 5 (a), the temperature difference 
between the hot He layer near the FW and the cold He layer on the side facing the breeding unit can reach a maximum of $21^{\circ} \mathrm{C}$. The temperature difference of flow channels with rectangular, finger or triangular fins are relatively small (Fig. 5 (b), (c) (d)). The thermal stress and strain in the FW structure can be effectively reduced by setting the fin structure on the wall surface facing plasma, which can transport cold helium from the side facing the breeding unit towards the main stream, preventing the hot helium layer from approaching the first wall surface loaded with high heat flux (see Fig.5 (c)).
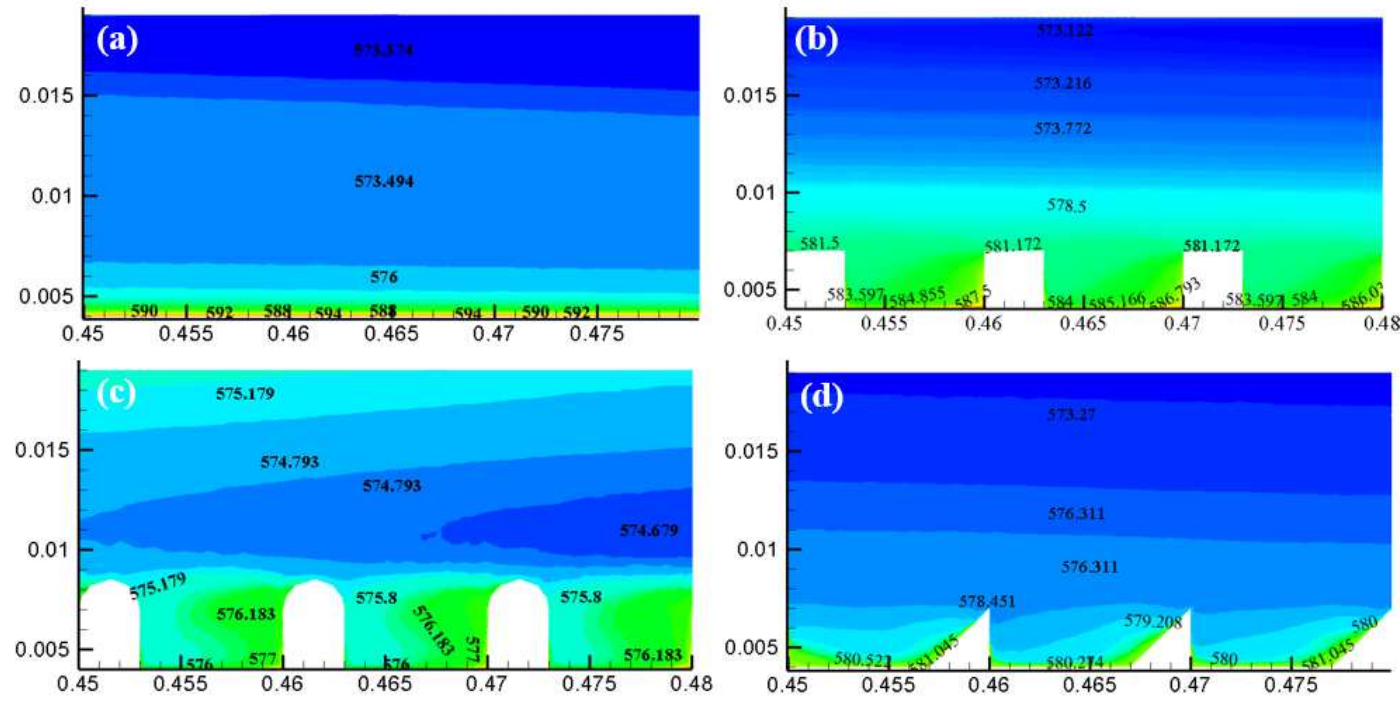

Fig.5 The temperature distribution of $\mathrm{He}$ on the cross section of the flow channel at 0.4 $\mathrm{MW} / \mathrm{m}^{2}$ when $\mathrm{G}=0.28 \mathrm{~kg} / \mathrm{s}, \mathrm{T}_{\text {in }}=573 \mathrm{~K}, \mathrm{P}=8 \mathrm{MPa}(\mathrm{x}=0, \mathrm{z}=0.45 \mathrm{~mm} \sim 0.48 \mathrm{~mm})(\mathrm{a})$ smooth flow channel (b) flow channel with rectangular fins (c)flow channel with finger fins (d) flow channel with triangular fins

Though the fin structure improves the temperature gradient of the FW, it also causes a larger pressure drop. So it is necessary to consider the influence of flow resistance on heat transfer. Fig. 6 shows the change of the average friction factor of different flow channels compared with the smooth flow channel and found that the fingers fins have 
the largest average friction factor and higher pressure drop. Considering economy and operating efficiency, the comprehensive heat transfer efficiency of fin structure flow channel with different FOM coefficients is used for evaluation. As shown in Fig7 (a), the fin structure provided on the inner surface of the FW can significantly increase the Nusselt number of the fluid, and the strong heat exchange capacity can be improved to a certain extent. However, at the same time, a substantial increase in the average friction factor due to the generated pressure drop, which lead to a smaller increase in the FOM coefficient (Fig. 7(b)).

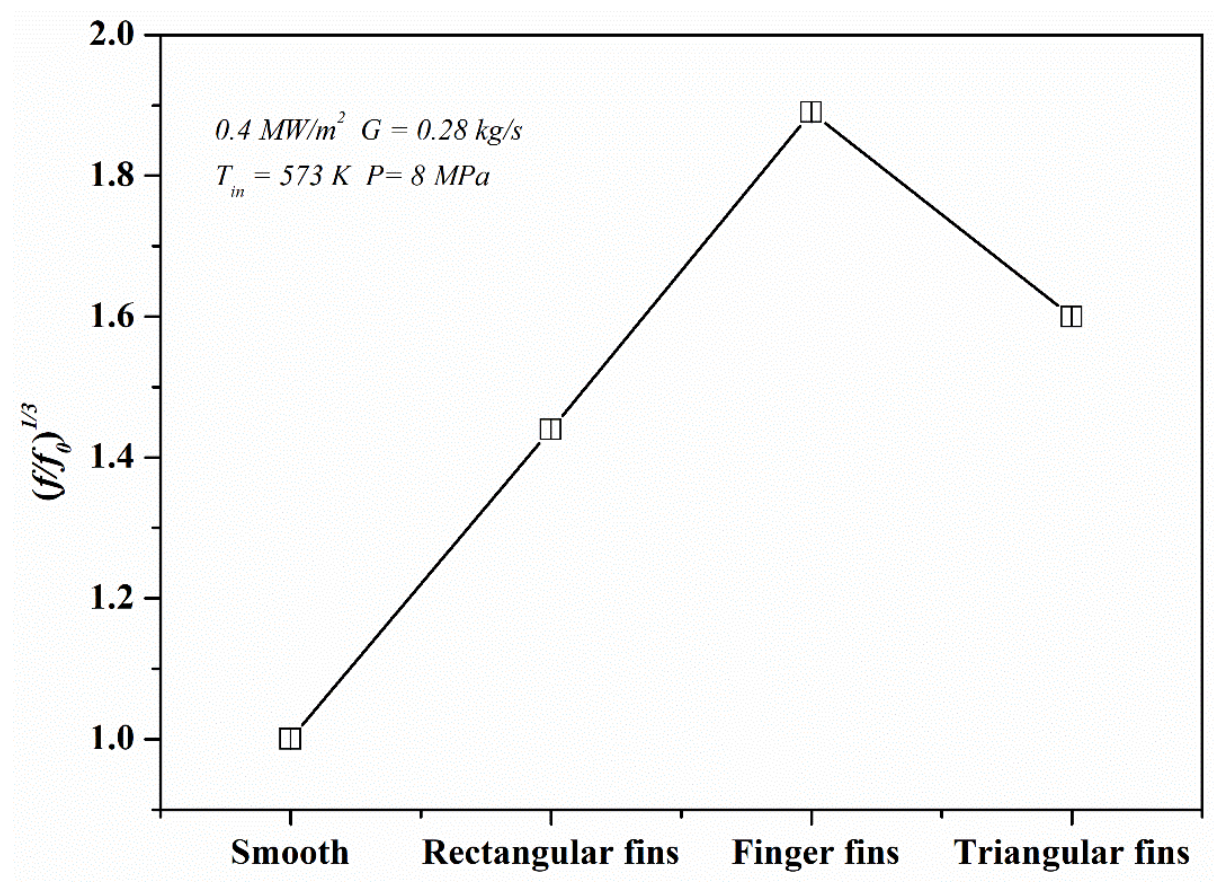

Fig. 6 The change of the average friction factor of different fin structure flow channel compared with that of a smooth flow channel 

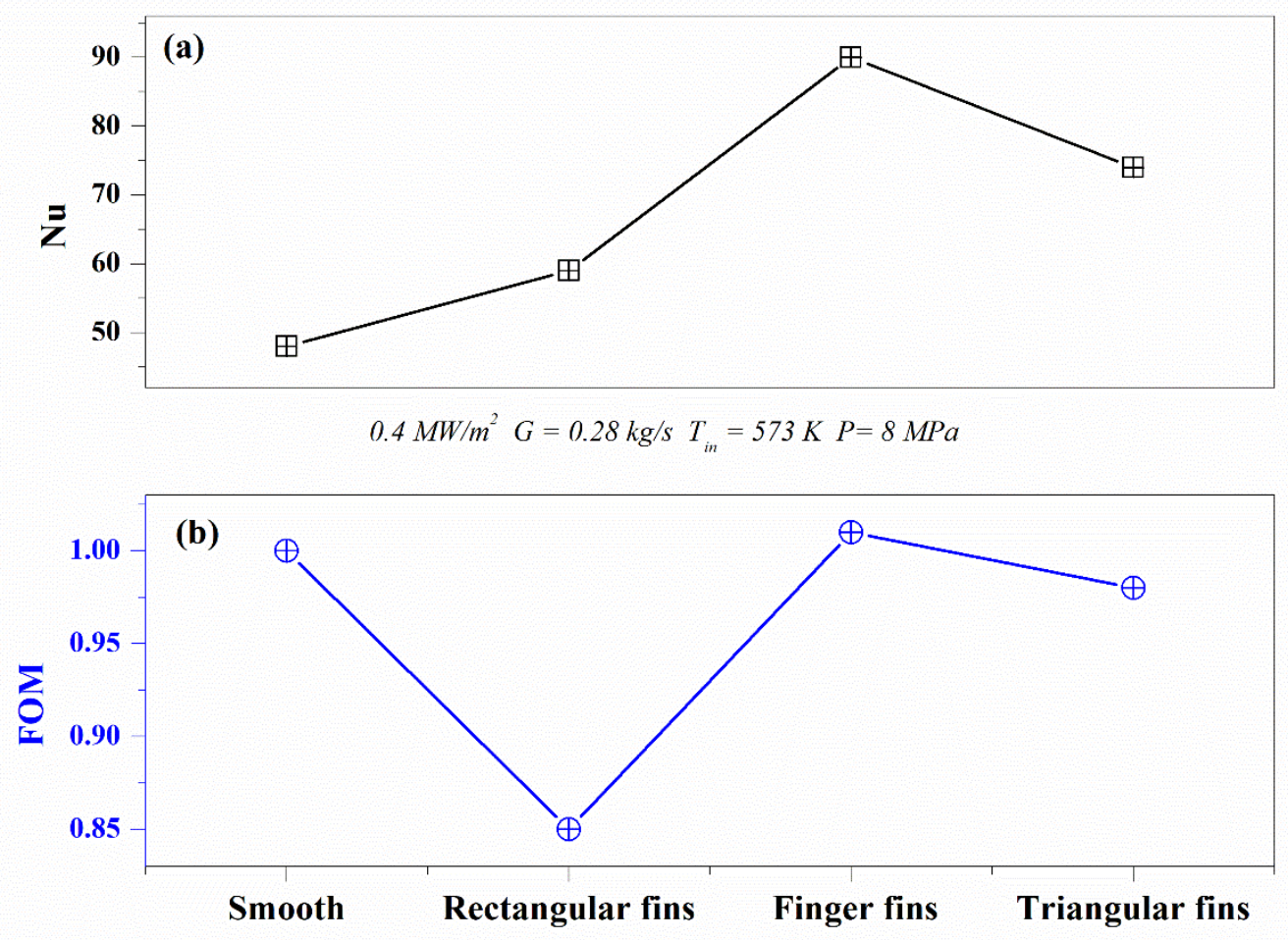

Fig. 7 (a) Nu numbers (b) FOM coefficients

In the early stage, the heat exchange capacity of the $\mathrm{He} / \mathrm{S}-\mathrm{CO}_{2}$ binary mixture gas and the pressure drop of the flow channel were analyzed. Comparing to the Pure $\mathrm{He}$ cooling, the mixture gas doped with $\mathrm{S}-\mathrm{CO}_{2}$ can significantly reduce the pressure drop of the flow channel. The fin structure improves the average Nusselt number to a certain extent; On the contrary, the pressure drop of the flow channel also increases. So, the overall comprehensive heat transfer efficiency tends to present improvements or even worse performance.

4.3 Cooperative heat transfer characteristics of $\mathrm{He} / \mathrm{S}-\mathrm{CO}_{2}$ binary mixture gas with fin structure

Fig 8 shows the velocity distribution and flow trajectory of the $\mathrm{He} / \mathrm{S}-\mathrm{CO}_{2}$ binary mixture gas at the cross section of the flow channel with three different fin structures. The fluid velocity in the center of the channel with three fin structures are significantly 
higher than that in the smooth channel, and the fluid velocity in the finger fins channel can reach $110 \mathrm{~m} / \mathrm{s}$ (Fig. $8(\mathrm{c}))$. The reason is that the fin structure increases the fluid disturbance and even produces eddy current to enhance the convective heat transfer.
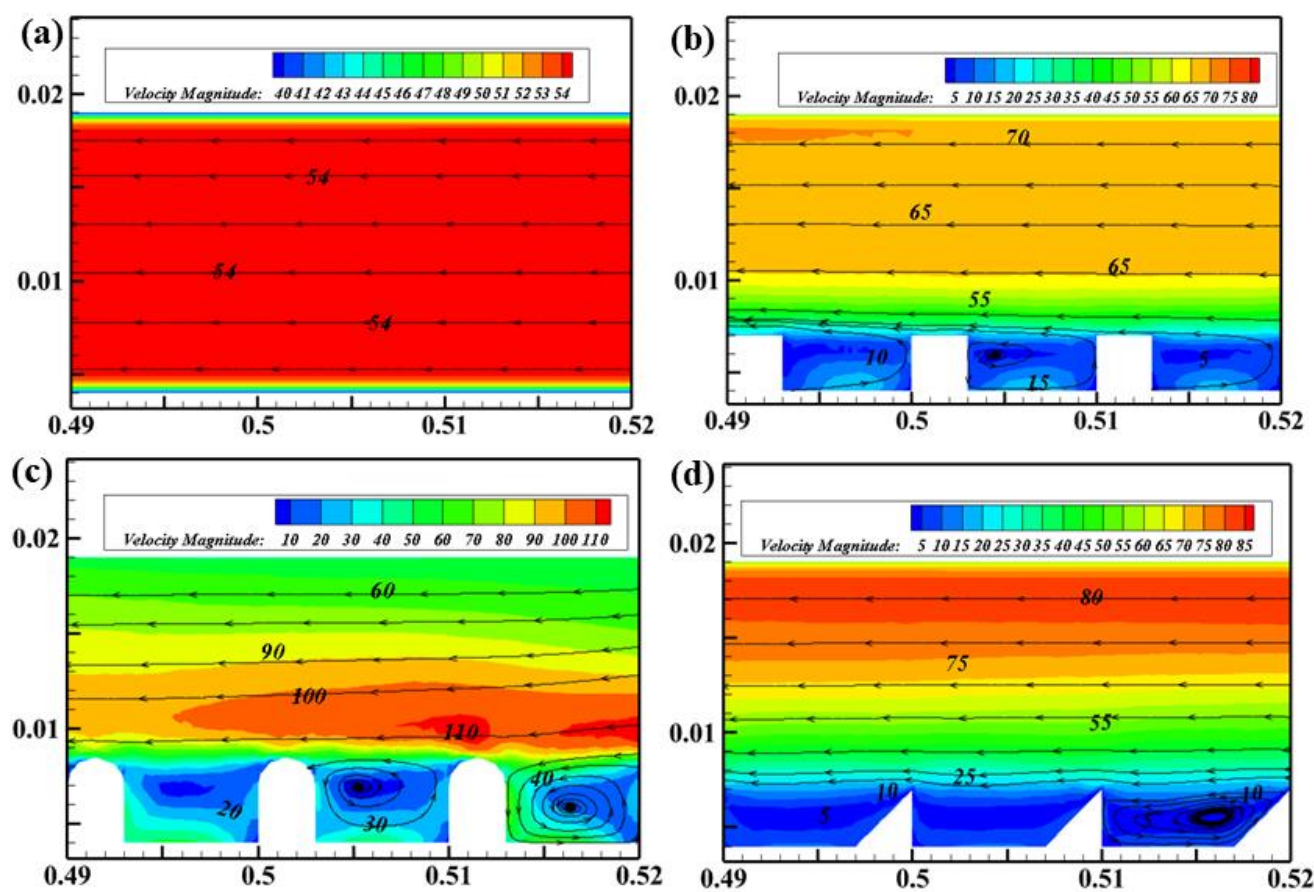

Fig. 8 Velocity cloud diagram of the center section of the flow channel with different fin structures $(\mathrm{z}=0.49 \sim 0.52 \mathrm{~mm})$

By monitoring the maximum temperature of the $\mathrm{FW}$ with different fin structures and the pressure drop of flow channel (as shown in Fig. 9), it is found that the maximum temperature gradually increases with increasing the volume ratio of $\mathrm{S}-\mathrm{CO}_{2}$ in the binary mixture gas. Setting different fin structures on the inner surface of FW can significantly reduce the maximum wall temperature, and the finger fins structure show the best effect. In addition, the pressure drop of the flow channel with fin structure is significantly higher than that of the smooth channel (Fig. 9 (b)). Due to the complex vortex formed near the wall surface, the flow velocity in the central fluid area increases, resulting in the increase 
of pressure drop. On the other hand, due to the high density of $\mathrm{S}-\mathrm{CO}_{2}$, at the same mass flow rate, the flow rate of binary mixture gas in the flow channel becomes smaller with the increase of $\mathrm{S}-\mathrm{CO}_{2}$ content, and the flowing pressure drop will be reduced accordingly.
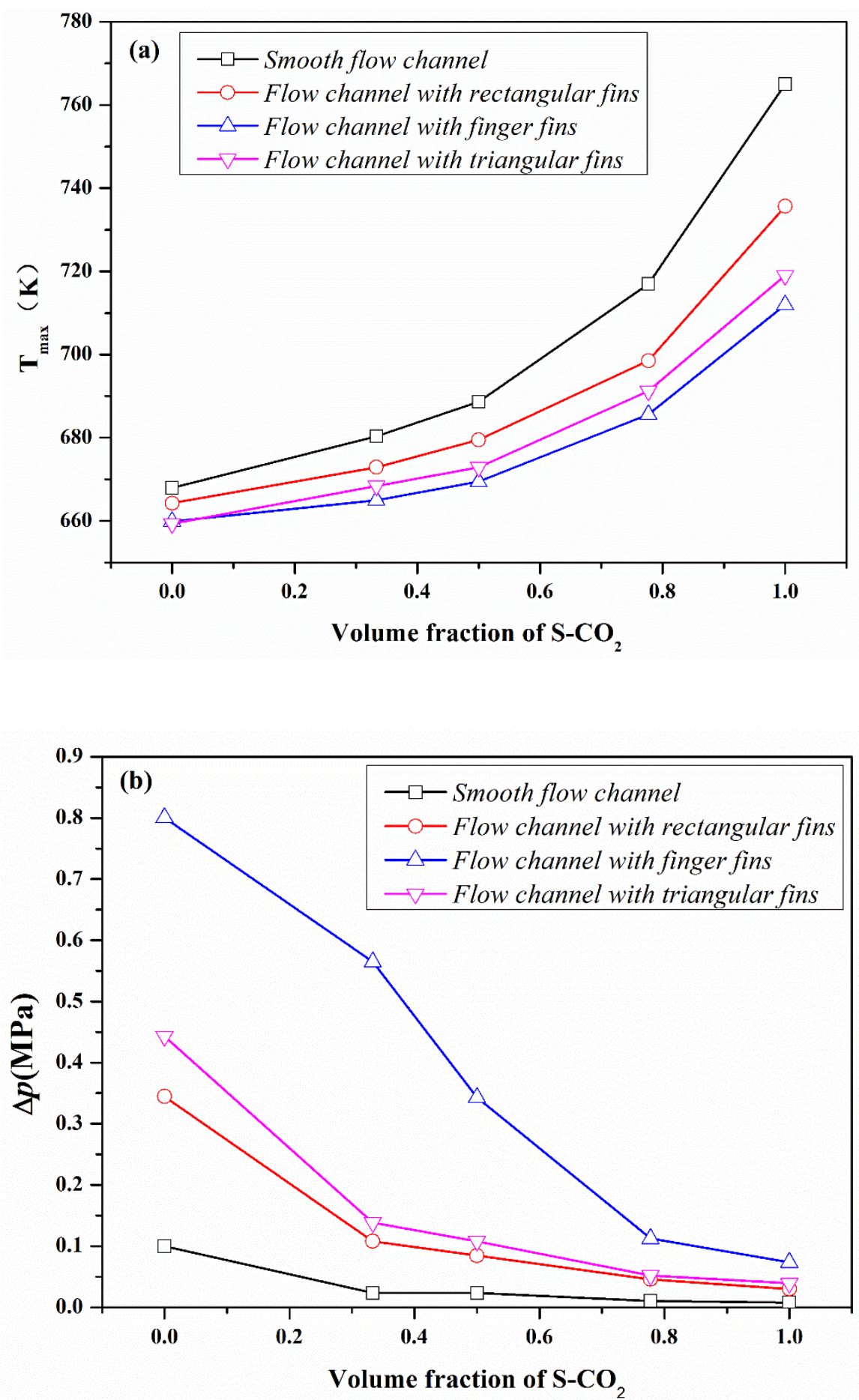

Fig. 9 (a) Maximum wall temperature; (b) Pressure drop in flow channel of $\mathrm{He} / \mathrm{S}-\mathrm{CO}_{2}$ 
binary mixture gas with different volume ratio

Fig. 10 shows the variation of the average Nusselt number of channel fluids with different fin structures with the volume fraction of $\mathrm{S}-\mathrm{CO}_{2}$. The simulation results show that with the increase of the volume fraction of $\mathrm{S}-\mathrm{CO}_{2}$, the Nu number of binary mixture gas increases first and then decreases. When the volume ratio of $\mathrm{S}-\mathrm{CO}_{2}$ to $\mathrm{He}$ is $2: 1$, the $\mathrm{Nu}$ number reaches the maximum and the convective heat transfer intensity of the fluid reaches the best. In addition, different fin structures have different gain effects on the convective heat transfer intensity of fluid. Comparing with the other two fin structures, the finger fin structure has the most obvious gain effect.

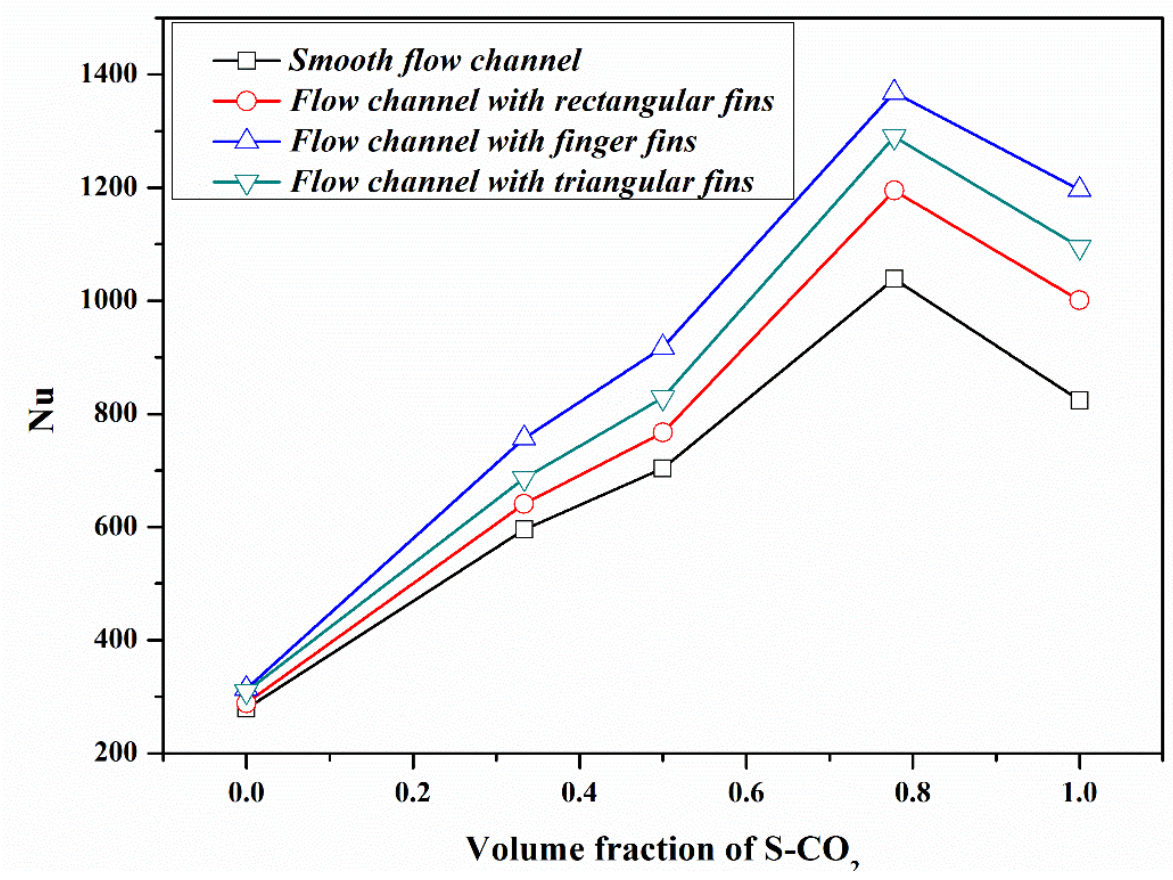

Fig. $10 \mathrm{Nu}$ number variation of binary mixture gas with $\mathrm{S}-\mathrm{CO}_{2}$ volume fraction

The previous analysis shows that different fin structures not only strengthen heat exchange, but also greatly enlarge the pressure drop of the flow channel, which lead to high cost. Therefore, FOM coefficient is introduced to evaluate the comprehensive heat transfer efficiency under different fin structures cooperating with binary mixture gas, as 
shown in Fig 11. With the increase of $\mathrm{S}-\mathrm{CO}_{2}$ volume fraction, the FOM coefficient increases first and then decreases and reaches the best at the volume ratio of $\mathrm{S}-\mathrm{CO}_{2}$ to helium is $2: 1$. On the other hand, the comprehensive heat transfer efficiency of triangular fins flow channel is better than that of finger fins and rectangular fins flow channel. The volume ratio of $\mathrm{S}-\mathrm{CO}_{2}$ to $\mathrm{He}$ is $2: 1$, and the $\mathrm{FOM}$ coefficient of triangular fin channel is increased by $310 \%$.

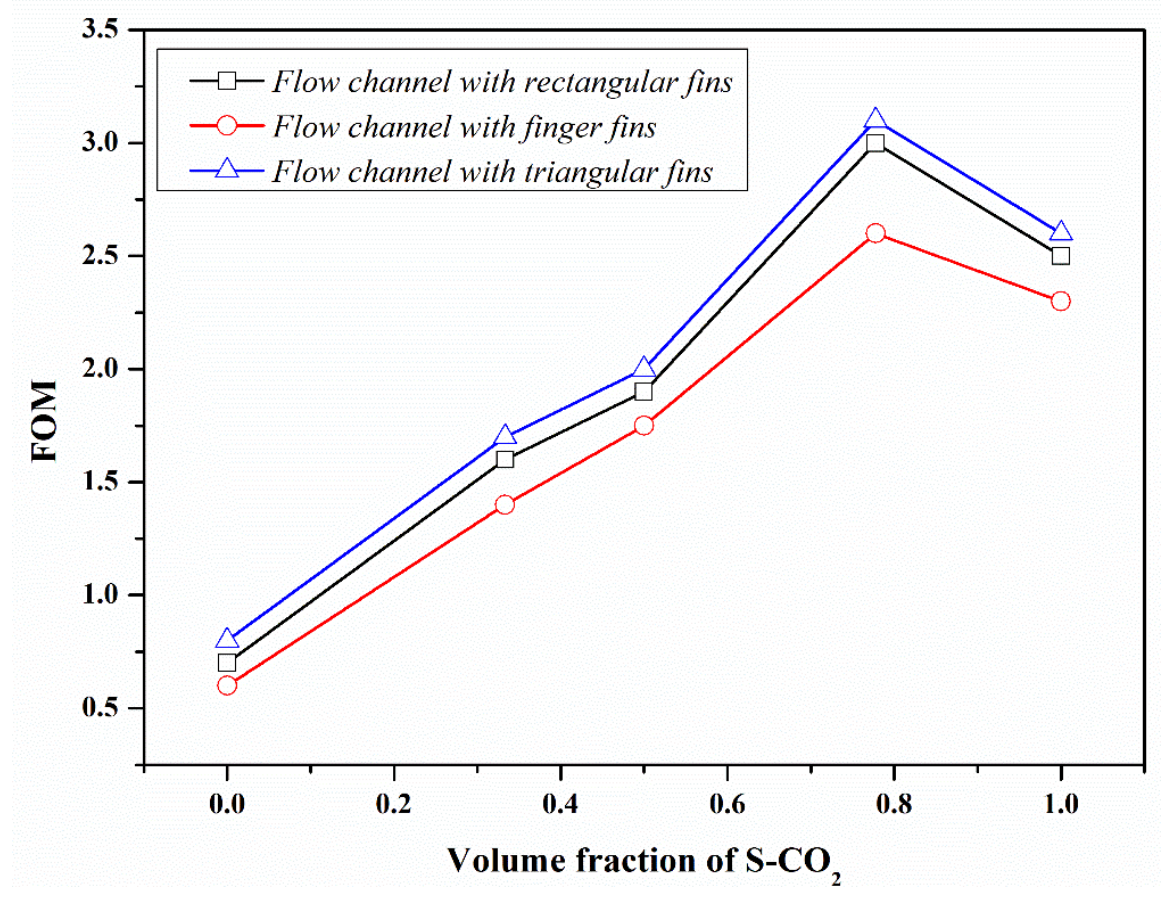

Fig. 11 Variation relationship between FOM coefficient and $\mathrm{S}-\mathrm{CO}_{2}$ volume fraction

\subsection{Parameter optimization of triangular fins flow channel}

Based on the optimization scheme of triangular fins flow channel for enhanced heat transfer, the effect of triangular fin pitch parameters on improving heat transfer efficiency needs to be further studied. Narrow fin pitch leads to complex manufacturing process and high manufacturing cost, while wide fin pitch causes $\mathrm{He} / \mathrm{S}-\mathrm{CO}_{2}$ binary mixture gas adheres to the flow channel surface and establishes a new boundary layer. In order to 
optimize the fin pitch, a three-dimensional numerical model is established by selecting different fin pitch under the condition of $\mathrm{S}-\mathrm{CO}_{2}$ to $\mathrm{He}$ volume ratio of 2:1.

Fig.12 show the variation of maximum surface temperature with different triangular fin pitch $(2,4,5,7,10 \mathrm{~mm})$ at $0.28 \mathrm{~kg} / \mathrm{s}$ inlet mass flow rate and condition of $\mathrm{S}-\mathrm{CO}_{2}$ to $\mathrm{He}$ volume ratio of 2:1. It can be observed from Fig. 12 that with the decrease of triangular fin pitch, the ability of withstand higher heat fluxes first increases and then decreases, and the maximum surface temperature reaches $692.2 \mathrm{k}$ at the pitch of $5 \mathrm{~mm}$. As the triangular fin pitch decreases from 5 to $2 \mathrm{~mm}$, its pressure drop decrease from 70 to $57.3 \mathrm{kPa}$. Whereas for fin pitch increases from $5 \mathrm{~mm}$ to $10 \mathrm{~mm}$ its pressure drop increase from 57.3 to $59.8 \mathrm{kPa}$.

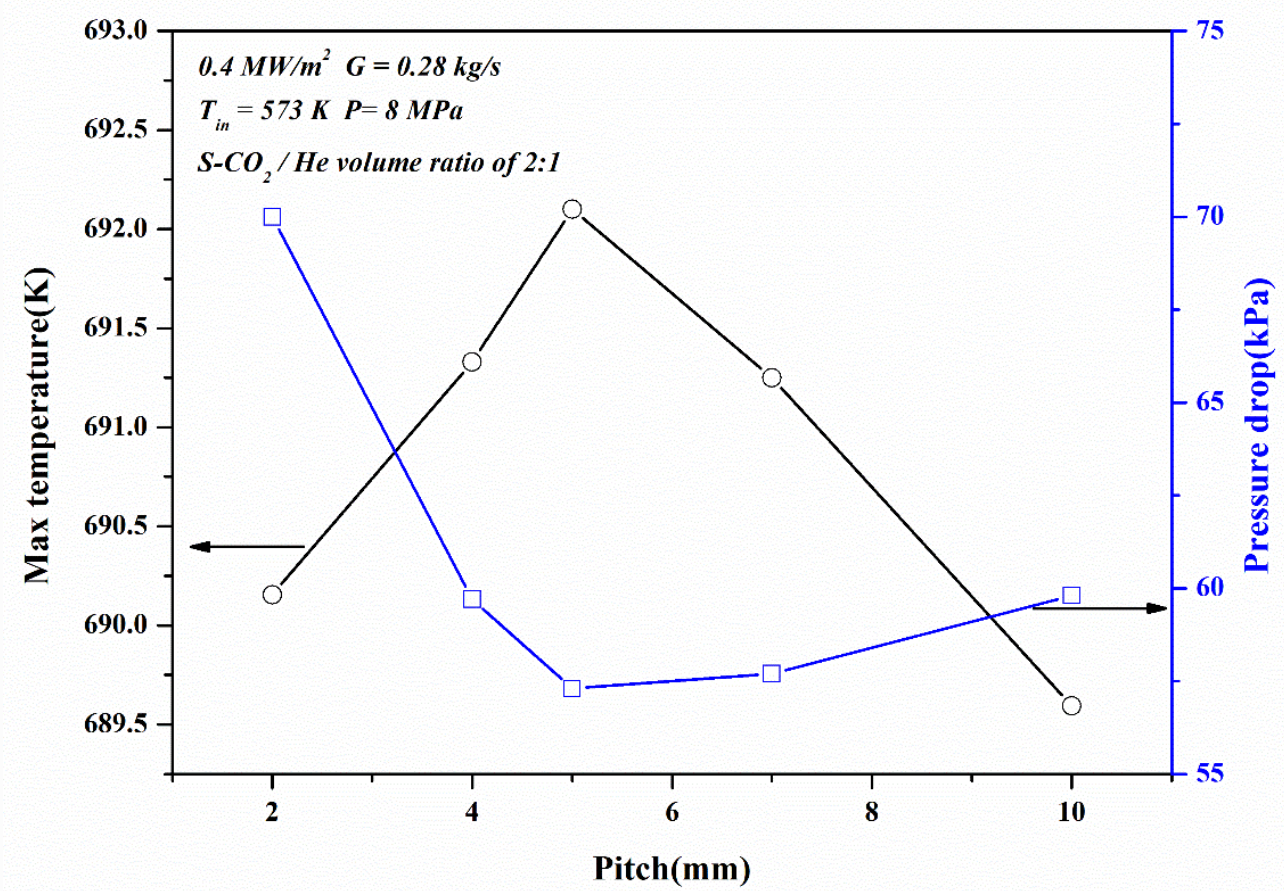

Fig.12. Comparison of maximum surface temperatures of FW with $0.28 \mathrm{~kg} / \mathrm{s}$ inlet mass flow rate at $\mathrm{S}-\mathrm{CO}_{2}$ to $\mathrm{He}$ volume ratio of 2:1 condition and 2 10 $\mathrm{mm}$ fin pitch.

Fig.13 shows $\mathrm{He} / \mathrm{S}-\mathrm{CO}_{2}$ gas velocity distribution $(\mathrm{m} / \mathrm{s})$ and flow track at cross 
sections of middle flow channels triangular with different fin pitches. It can be observed from Fig13(a) that obvious stable boundary layer and clear flow hierarchy can be seen near the wall surface of the smooth flow channel. Fig13(b) shows the flow channel with triangular fin, in which the boundary layer is destroyed and local disturbances is formed between the fins under the condition of narrow fin pitch. It is evident from Fig.13 (b)-(f) that as the distance between fins becomes wider, $\mathrm{He} / \mathrm{S}-\mathrm{CO}_{2}$ binary mixture gas gradually adheres to the wall surface between fins to establish a new boundary layer. The optimization parameter of triangular fins should be between $7 \sim 10 \mathrm{~mm}$.
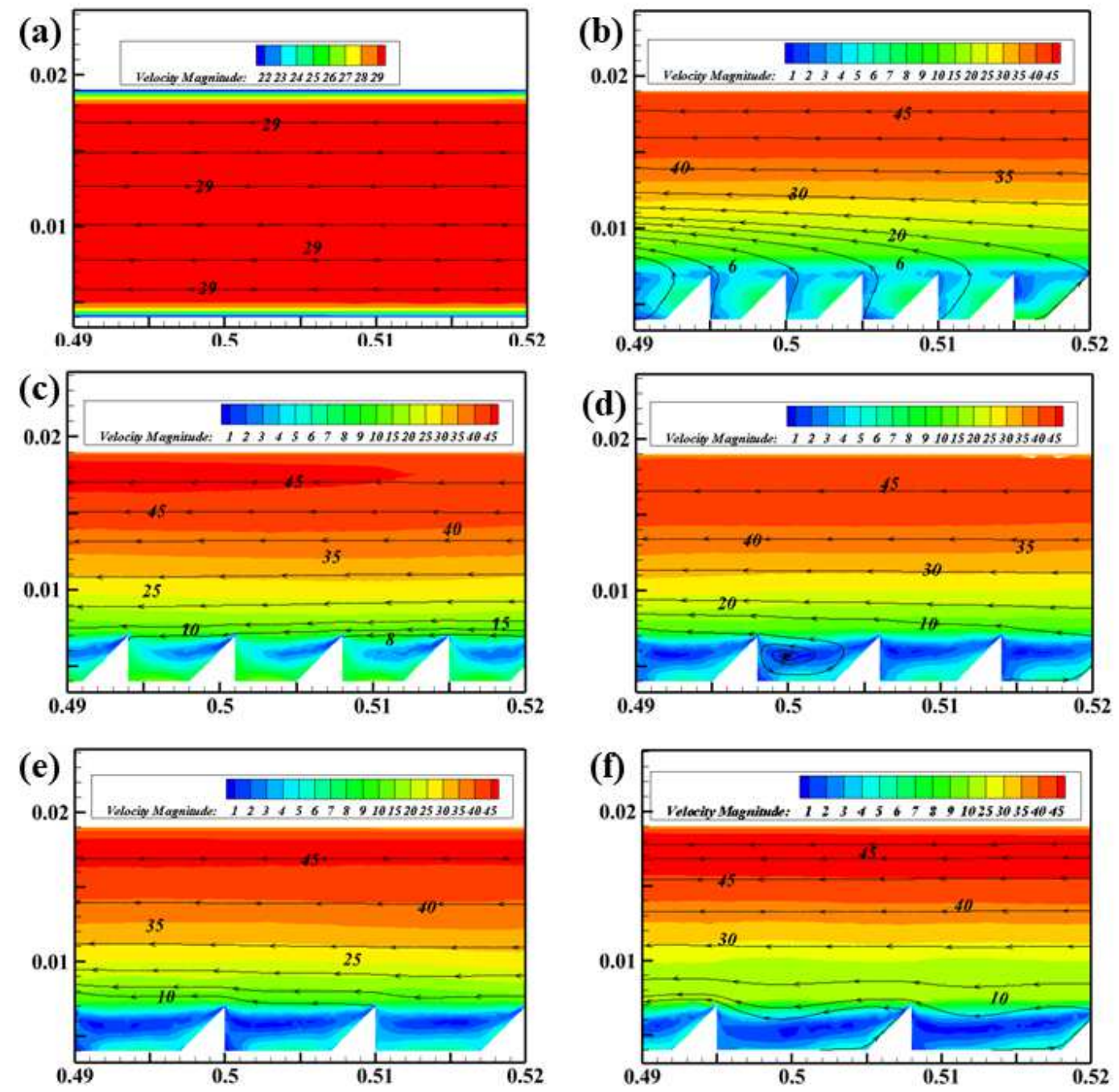

Fig.13 He / S-CO 2 gas velocity distribution $(\mathrm{m} / \mathrm{s})$ and flow track at cross sections of 
middle flow channels with different triangular fin pitches: (a)smooth flow channel; (b)channel with triangular fins with fin pitch of $2 \mathrm{~mm}$; (c) channel with triangular fins with fin pitch of 4mm; (d) channel with triangular fins with fin pitch of 5mm; (e) channel with triangular fins with fin pitch of $7 \mathrm{~mm}$; (f) channel with triangular fins with fin pitch of $10 \mathrm{~mm}$.

\section{Conclusion}

In this paper, the cooling performance of $\mathrm{He} / \mathrm{S}-\mathrm{CO}_{2}$ binary mixture gas with different volume ratio and fin structure were simulated by ANSYS / CFD method. The main conclusions are as follows:

1. The maximum temperature of the FW gradually increases with the increase of volume fraction of $\mathrm{S}-\mathrm{CO}_{2}$, and the variation tendencies of pressure drops in flow channels are completely opposite.

2. The results show that fin structures change the flow direction of the fluid near the wall, increase the turbulent kinetic energy of the fluid, form eddy current and enhance the heat transfer performance. It can be observed that compared with smooth channel, channel with fins can significantly improve the convective heat transfer intensity and the Nu number of flow channel with finger fins is increased by $80 \%$. However, the average friction coefficient of finger fins flow channel is greatly improved, resulting in that the FOM coefficient is much than that of the smooth flow channel. 
3. The synergistic performance of $\mathrm{He} / \mathrm{S}-\mathrm{CO}_{2}$ binary mixture gas with heat transfer enhancement structure (fins) is comprehensively evaluated. It can be observed that with the increase of the volume fraction of $\mathrm{S}-\mathrm{CO}_{2}$, the FOM coefficient increases first and then decreases. When the volume ratio of $\mathrm{S}-\mathrm{CO}_{2}$ to helium is $2: 1$, the comprehensive heat transfer performance reaches the best. Compared with the smooth flow channel, the FOM coefficient of triangular fin channel is increased by $310 \%$.

4. The effect of triangular fins pitch parameters on improving heat transfer efficiency is further studied. The result shows with the distance between fins becomes wider, $\mathrm{He} / \mathrm{S}-\mathrm{CO}_{2}$ binary mixture gas gradually adheres to the surface wall between fins to establish a new boundary layer. The optimization parameter of triangular fins should be between $7 \sim 10 \mathrm{~mm}$.

\section{Acknowledgement}

This work was supported by the National Natural Science Foundation of China under Grant 11975022 and in part by the National Magnetic Confinement Fusion Program under Grant2017YFE0300603, Grant2018YFE0309100, and Grant2015GB120006.

\section{Reference:}

[1]. Wang, X. Y., et al., Current design and R\&D progress of the Chinese helium cooled ceramic breeder test blanket system, Nuclear Fusion 59.7 (2019): 076019.doi: 10.1088/1741-4326/ab0c32.

[2]. Shin C W, Lee E H, Kim S K, et al., Design and experimental study of 
adsorption bed for the helium coolant purification system, Fusion Engineering and Design, 2020, 155: 111687.doi: 10.1016/j.fusengdes.2020.111687.

[3]. Zhao H, Peterson P F., Multiple reheat helium Brayton cycles for sodium cooled fast reactors, Nuclear Engineering and Design, 2008, 238(7): 15351546.doi: 10.1016/j.nucengdes.2007.12.002.

[4]. Eter A, Groeneveld D, Tavoularis S., Convective heat transfer in supercritical flows of $\mathrm{CO}_{2}$ in tubes with and without flow obstacles, Nuclear Engineering and Design, 2017, 313: 162-176.doi: 10.1016/j.nucengdes.2016.12.016.

[5]. Cheng L, Ribatski G, Thome J R., Analysis of supercritical CO2 cooling in macro-and micro-channels, International journal of refrigeration, 2008, 31(8): 1301-1316.doi: 10.1016/j.ijrefrig.2008.01.010.

[6]. Kato Y, Nitawaki T, Muto Y., Medium temperature carbon dioxide gas turbine reactor, Nuclear Engineering and Design, 2004, 230(1-3): 195-207.doi: 10.1016/j.nucengdes.2003.12.002.

[7]. Jeong W S, Lee J I, Jeong Y H., Potential improvements of supercritical recompression $\mathrm{CO}_{2}$ Brayton cycle by mixing other gases for power conversion system of a SFR, Nuclear Engineering and Design, 2011, 241(6): 21282137.doi: 10.1016/j.nucengdes.2011.03.043.

[8]. Cheng D, Wang W, Deng H, et al., Numerical investigation of channel for helium cooled China HCCB-TBM first wall by application enhance heat transfer method, Journal of Fusion Energy, 2016, 35(5): 748-757.doi: 10.1007/s10894-016-0098-6. 
[9]. Andreozzi A, Manca O, Nardini S, et al., Forced convection enhancement in channels with transversal fins and nanofluids, Applied Thermal Engineering, 2016, 98: 1044-1053.doi: 10.1016/j.applthermaleng.2015.12.140.

[10]. Hou F, Chang H, Zhao Y, et al., Experimental study of critical heat flux enhancement with hypervapotron structure under natural circulation conditions, Nuclear Engineering and Design, 2017, 316: 209-217.doi: 10.1016/j.nucengdes.2017.03.013.

[11]. Kanti P K, Sharma K V, Said Z, et al., Experimental investigation on thermohydraulic performance of water-based fly ash-Cu hybrid nanofluid flow in a pipe at various inlet fluid temperatures, International Communications in Heat and Mass $\quad$ Transfer, 2021, 124: 105238.doi: 10.1016/j.icheatmasstransfer.2021.105238.

[12]. Ali A M, Rona A, Kadhim H T, et al., Thermo-hydraulic performance of a circular microchannel heat sink using swirl flow and nanofluid, Applied Thermal Engineering, 2021, 191: 116817.doi: 10.1016/j.applthermaleng.2021.116817. 